\title{
Hindfoot Infections: What to do and How?
}

\author{
${ }^{1}$ Nirmal Raj Gopinathan, ${ }^{2}$ Mandeep Singh Dhillon, ${ }^{3}$ B alaji Saibaba
}

\begin{abstract}
Hindfoot infections though uncommon, pose a major cause of orthopedic concern in view of their delayed diagnosis and lack of adequate expertise in their successful management. If untreated, these infections can cause significant morbidity and functional impairment which can even culminate in amputation. Successful management of this otherwise disabling condition warrants a thorough knowledge about the various etiopathological factors, the myriad clinical presentations, available diagnostic modalities and specific management strategies-medical and/or surgical. Timely diagnosis and appropriate intervention is the key for eradicating hindfoot infections and restoring the patient's functional status.
\end{abstract}

Keywords: Infection, Hindfoot, Calcaneum, Talus, Management.

How to cite this article: Gopinathan NR, Dhillon MS, Saibaba B. Hindfoot Infections: What to do and How? J Foot Ankle Surg (Asia-Pacific) 2015;2(1):31-37.

\section{Source of support $\mathrm{Nil}$}

Conflict of interest None

\section{INTRODUCTION}

Infections of the calcaneus and talus, though not as common as that of long bones and vertebrae, are equally devastating and can place the clinician in a diagnostic and therapeutic dilemma. ${ }^{1}$ Calcaneus serves as the point of contact of the body with the ground. The importance in discussing these conditions lies in the fact that the diagnosis is often delayed, resulting in chronic morbidity, which significantly affects the quality of life and adds to the economic burden to the individual and society. Apart from pyogenic infections, tuberculosis $^{2}$ and fungal ${ }^{3}$ infections are also seen, especially in immunocompromised and diabetic patients adding to their morbidity. In the following review we have tried to comprehensively review the available clinical literature regarding these morbid conditions and their management in brief.

\footnotetext{
${ }^{1}$ Assistant Professor, ${ }^{2}$ P rofessor and Head ${ }^{3}$ Senior Resident

${ }^{1-3}$ Department of Orthopedics, P ostgraduate Institute of Medical Education and Research, Chandigarh, India
}

Corresponding Author: Mandeep Singh Dhillon, Professor and Head, Department of Orthopedics, Postgraduate Institute of Medical Education and Research, Chandigarh, India, Phone: 919815951090, e-mail: drdhillon@gmail.com

\section{VULNERABILITY TO INFECTIONS}

The limited muscle mass surrounding the bones of the hindfoot, the precarious vascularity which can be compromised easily and importantly the role of these bones in transmission of body weight dictate the vulnerability of the hindfoot to trauma and infections. Additionally the reduced skin sensation resulting from neuropathy related to diabetes and other conditions increases the vulnerability of these bones in getting infected, especially when these patients develop trophic foot ulcers. Unique to the Asian and African scenario, where barefoot walking is common, thorn pricks, insect bites and other minor penetrating injuries become a significant predisposing factor for hind foot infections and have to be considered in all differential diagnoses. ${ }^{4}$

\section{DIAGNOSTIC MODALITIES}

The most important factor which improves the management of these conditions is fact that the clinician should be able to pick up the symptoms and signs at the earliest with vigil. As for all infections, leukocytosis, elevated erythrocyte sedimentation rate and elevated levels of C-reactive proteins are corroborative. Additionally, lymphocytosis may be seen in tuberculosis. Local X-rays can define the bony extent of the lesion when osteomyelitis develops, but in the early phases improved imaging modalities like computed tomography (CT), magnetic resonance imaging (MRI) and nuclear bone scan can be of significant help (Figs 1 and 2).

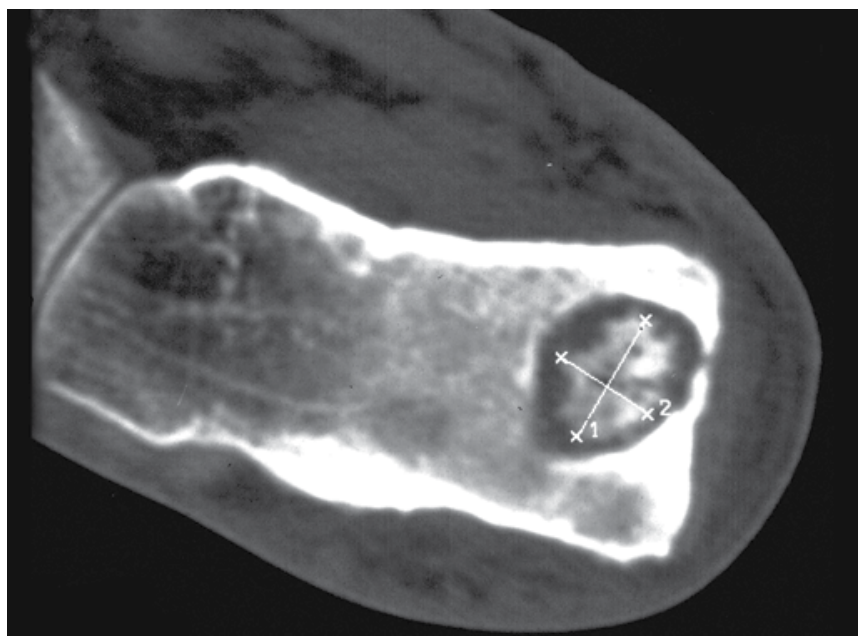

Fig. 1: Computed tomography scan showing well-defined sequestrum in calcaneus 


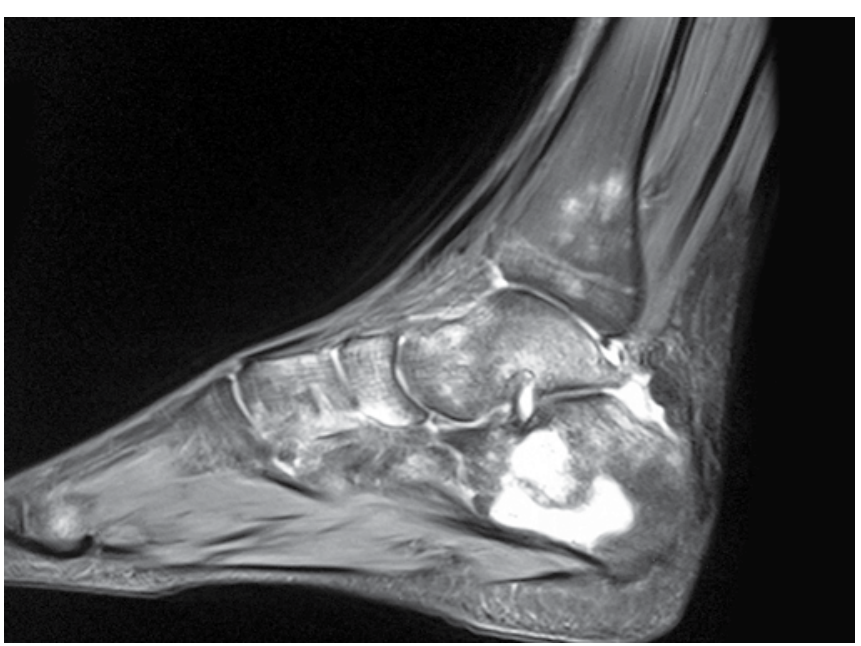

Fig. 2: Magnetic resonance imaging showing bony TB in pre-destructive phase

\section{TYPES OF INFECTIONS}

1. Pyogenic: Among the various etiologies known to cause hindfoot osteomyelitis, pyogenic infections top the chart ahead of tubercular and fungal infections. In children hematogenous spread of infection to calcaneum has also been frequently described. ${ }^{5}$ The clinician should be vigilant and must give weightage to the child's complaint of inability to bear weight accompanied by fever for a timely diagnosis. Staphylococcus is the most commonly isolated organism as usual, but a series of clinical case reports have termed a variety of Gram-positive, Gram-negative and anaerobic etiology under various circumstances. ${ }^{1,6}$ No specific age group is defined more susceptible, although neurogenic ulcers due to diabetes affect middle and old aged individuals more commonly.

2. Post-traumatic osteomyelitis: The most common reason for pyogenic osteomyelitis is trauma, followed by other conditions like surgical interventions, especially in neuropathic feet. The relatively thin soft tissue cover is vulnerable to trauma and frequently breaks down after ill-timed surgical interventions. This results in exposure of fracture surfaces to external environment and onset of osteomyelitis (Figs 3 and 4). Merlet et al in their observational study in 42 patients with calcaneal osteomyelitis found out that $62 \%$ of their patients had post-traumatic osteomyelitis and 38\% were secondary to neurological damage. ${ }^{1}$ Gale and Scott in their report warned that a puncture wound of the foot with persistent pain should alert the
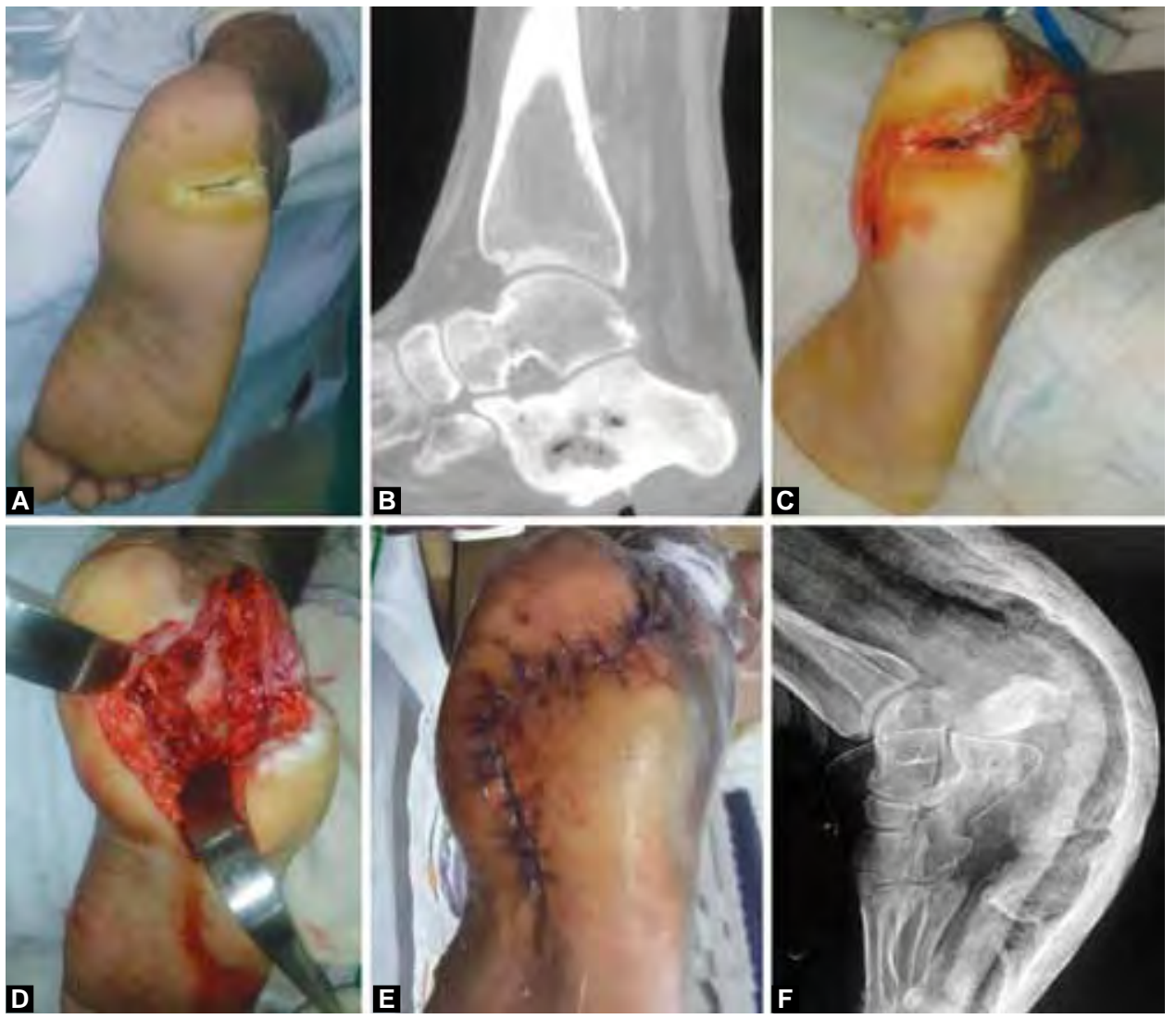

Figs 3A to F: Post-traumatic osteomyelitis: (A)Preoperative clinical photograph showing a discharging wound on the plantar surface of heel, (B) computed tomography scan showing the presence of a cavity within the calcaneus, (C) intraoperative photograph showing the utilization of a modified skin incision, (D) intraoperative photograph showing the calcaneal cavity after thorough debridement, (E) postoperative clinical photograph showing adequate skin closure and preservation of heel pad and $(F)$ postoperative radiograph after satisfactory calcaneal salvage 


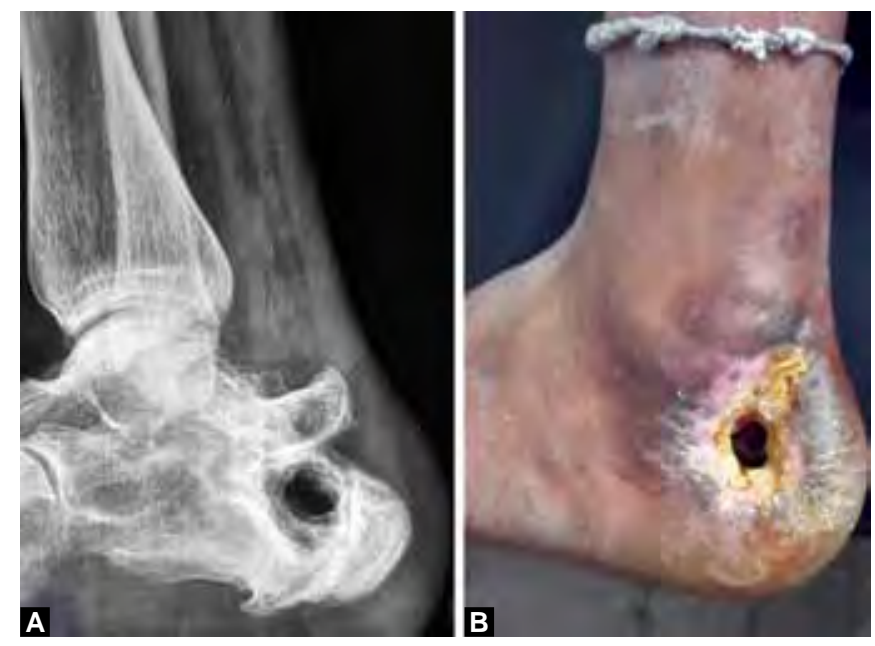

Figs 4A and B: Clinical (A) and radiographic (B) images depicting the persistence of infection due to the presence of nonhealing cavity within the calcaneus

clinician of a Pseudomonas aeruginosa osteomyelitis. ${ }^{7}$ In fact, Yüksel reported a case of calcaneal osteomyelitis in a newborn child following the Guthrie test (heel prick test) done for screening phenylketonuria. ${ }^{8}$ Wronka reported a case of calcaneal osteomyelitis following steroid infiltration for plantar fasciitis. ${ }^{9}$

3. Post surgical infections: Apart from contaminated open fractures, pyogenic infections following surgical interventions form a major chunk of preventable hindfoot osteomyelitis cases. The skin and soft tissues

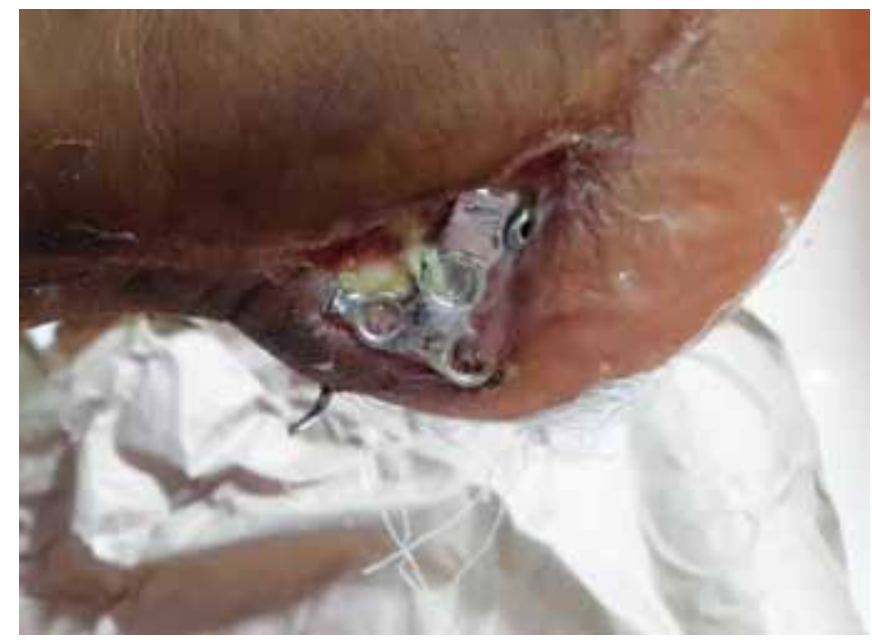

Fig. 5: Delayed post surgical infection presenting as exposed calcaneal implant after fracture fixation

are vulnerable to break down and subsequent infection if surgical approaches are not properly planned in time and site (Fig. 6B). The postsurgical infection can present early or late (Fig. 5). Poor knowledge of the possible devastating outcome and poor surgical technique for the management of calcaneal fractures still remains a major etiological factor for hindfoot infections.

4. Foreign body induced infections: Foreign bodies have been known to cause various types of reactions in
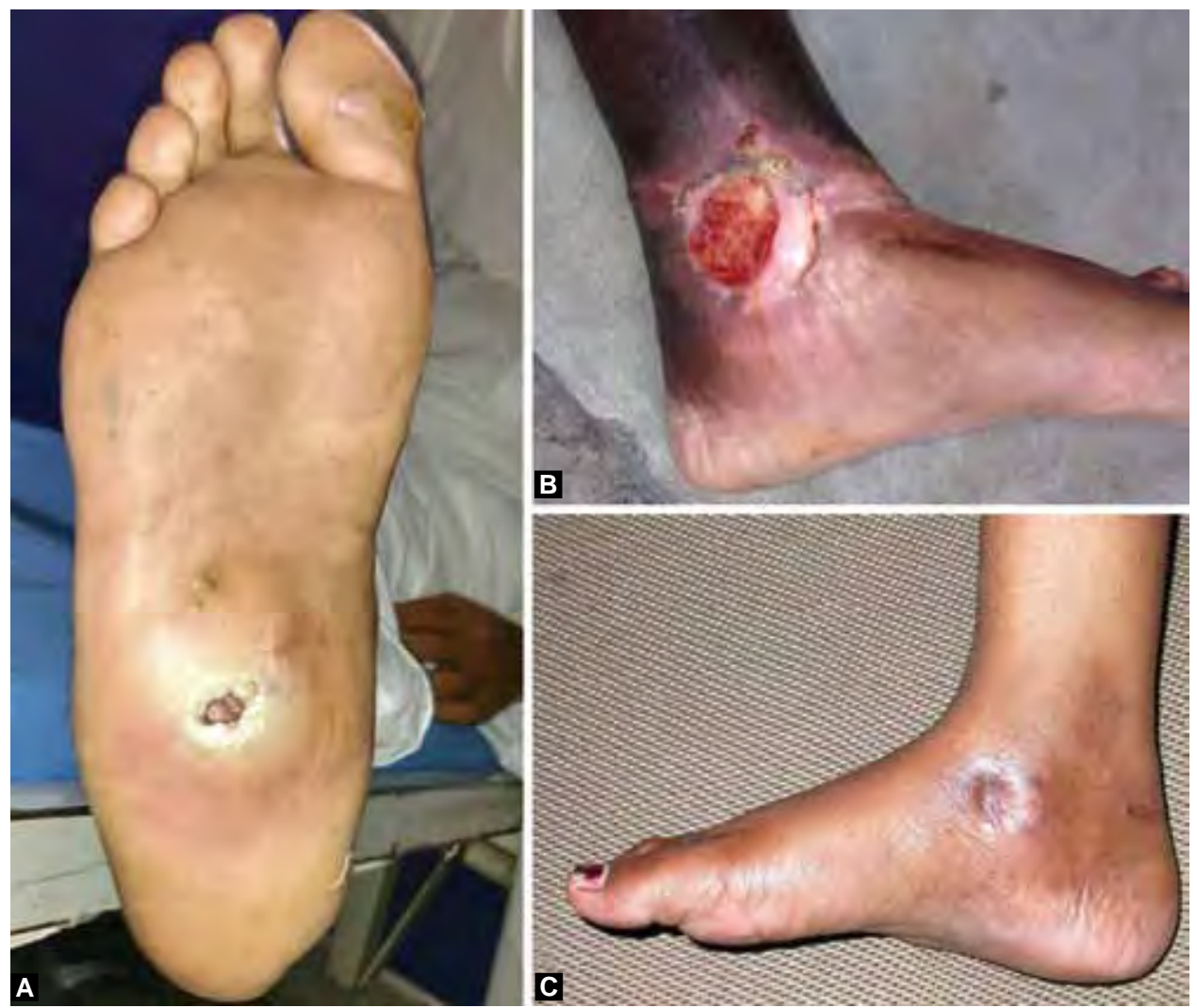

Figs 6A to C: Different types of ulcers presenting in the foot and ankle: (A) Nonhealing plantar ulcer secondary to retained foreign body (thorn), (B) post-traumatic ulcer on the medial aspect of ankle and $(C)$ clinical photograph showing tubercular ulcer on the medial aspect of hind foot 
the body, and in the foot, foreign bodies can easily penetrate and cause unusual presentations of lowgrade infections. The foot is a common site of puncture wounds, especially in underdeveloped countries where a lot of people still walk barefoot. These pricking injuries are usually considered trivial and not given much thought initially, and could present with a non-healing infection. In the foot, the commonest foreign body to produce this type of pathological reaction is a thorn (Fig. 6A) or a wooden splinter, and that too is very rare. ${ }^{10}$

Most foreign bodies are radiopaque substances and are easily detected on standard X-rays, when associated with a specific history, and a persistent discharge, ${ }^{11}$ some materials like wood or plastic cause problems in diagnosis and avoidable delays in appropriate treatment. However in some cases ${ }^{10}$ an early and accurate diagnosis minimizes morbidity, and perhaps avoids excessive surgery. Most organic materials, when embedded in bone, do not produce a significant radiologically discernable reaction, but some may mimic changes those suggest a bone tumor or infection. Hence, different modalities have been tried in an attempt to establish the diagnosis. Perhaps the cheapest and most convenient method is ultrasonography, used successfully for as small a fragment as a $0.5 \mathrm{~mm}$ thick thorn. This has the added advantage that it can be used as an aid even in the operating environment. Both CT and MRI are also excellent modalities, but, with the disadvantage of economical constraints and limited availability in poorer countries.

5. Tubercular infections: Tuberculosis of the foot and ankle is an uncommon presentation of skeletal tuberculosis (Fig. 6C). The uncommon site, lack of awareness, and ability to mimic other disorders clinically and on radiographs, leads to diagnostic and therapeutic delays. In the early stages and when the disease is limited to bone, medical treatment leads to excellent healing and limited residual disabilities. Joint involvement occurs because of spread from a periarticular bony focus, leading to a stiff foot and residual deformities. In a previous study, the senior author (MSD) reviewed 74 cases foot and ankle tuberculosis and found calcaneus to be the most commonly affected bone. ${ }^{12}$

The most common radiologic finding is that of osteoporosis, which may be intense. Cancellous bone involvement may present as a cystic lesion with or without sequestrum (Figs 7A and B). In another review by the same senior author (MSD) ${ }_{1}^{13}$ out of a series of 92 cases of foot $\mathrm{TB}$, osteolytic variety was reported in 11 calcaneus. Computed tomography scans and magnetic resonance imaging are helpful examinations. Because the disease is paucibacillary, a positive acid fast bacilli culture is rare and the diagnosis usually is confirmed by obtaining granulomatous tissue on biopsy. The treatment basically is medical, with surgical intervention being reserved for patients with intractable disease or as a salvage procedure for patients with deformed hindfoot joints. Even after many years after completion of anti-tubercular treatment, the radiographic residua of a cystic cavity may persist.

6. Fungal infections: Although uncommon, fungal infections of the calcaneus and hindfoot have been reported in literature (Figs $8 \mathrm{~A}$ to $\mathrm{C}$ ). The spectrum of infection varies from common Candida infections to extremely rare pathogens. Primary mycetoma presents with tumefaction, multiple discharging sinuses and grain filled pus. ${ }^{14}$ Pennekamp et al ${ }^{15}$ described a case of scedosporiosis of calcaneus following an open wound which ultimately led to a fatal outcome due to disseminated spread including cerebrum. Apart from routine investigative modality, special fungal stains and culture techniques are to be utilized in confirming the diagnosis when suspected.

The basis of management remains adequate surgical debridement and recent novel antifungal drugs have made limb salvage possible. Cetrulo et $\mathrm{al}^{16}$ reported a case of Scedosporium inflatum osteomyelitis
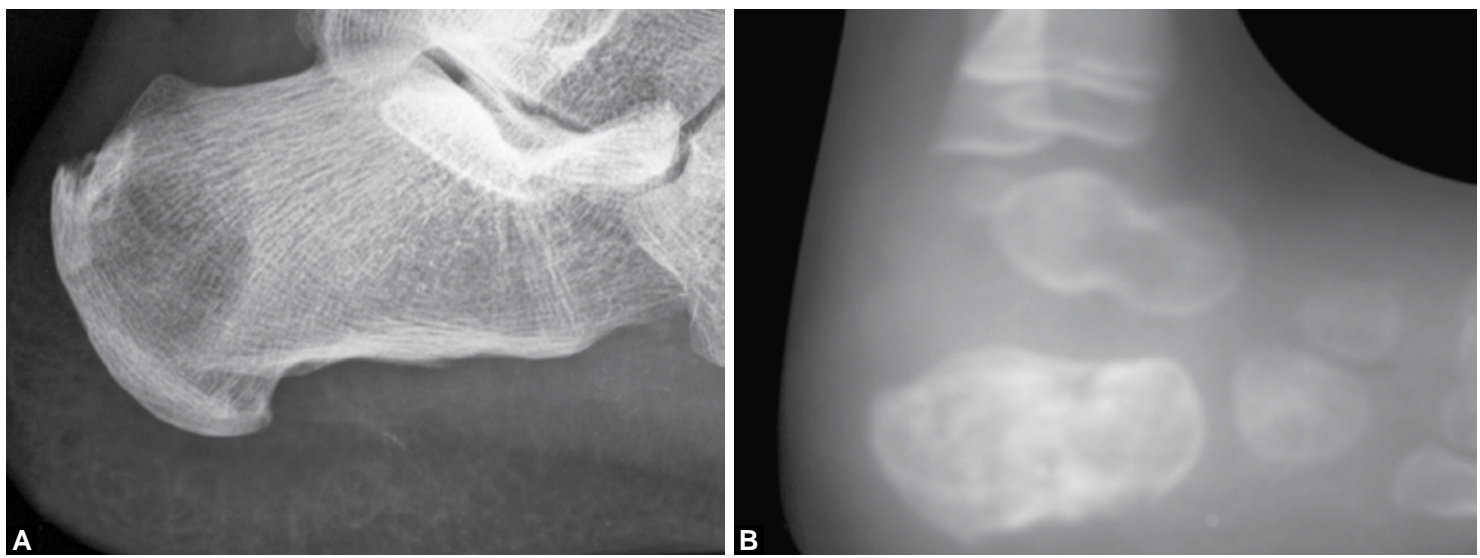

Figs 7A and B: Radiograph demonstrating TB as a destructive lesion in an adult (A) and in a child (B) 

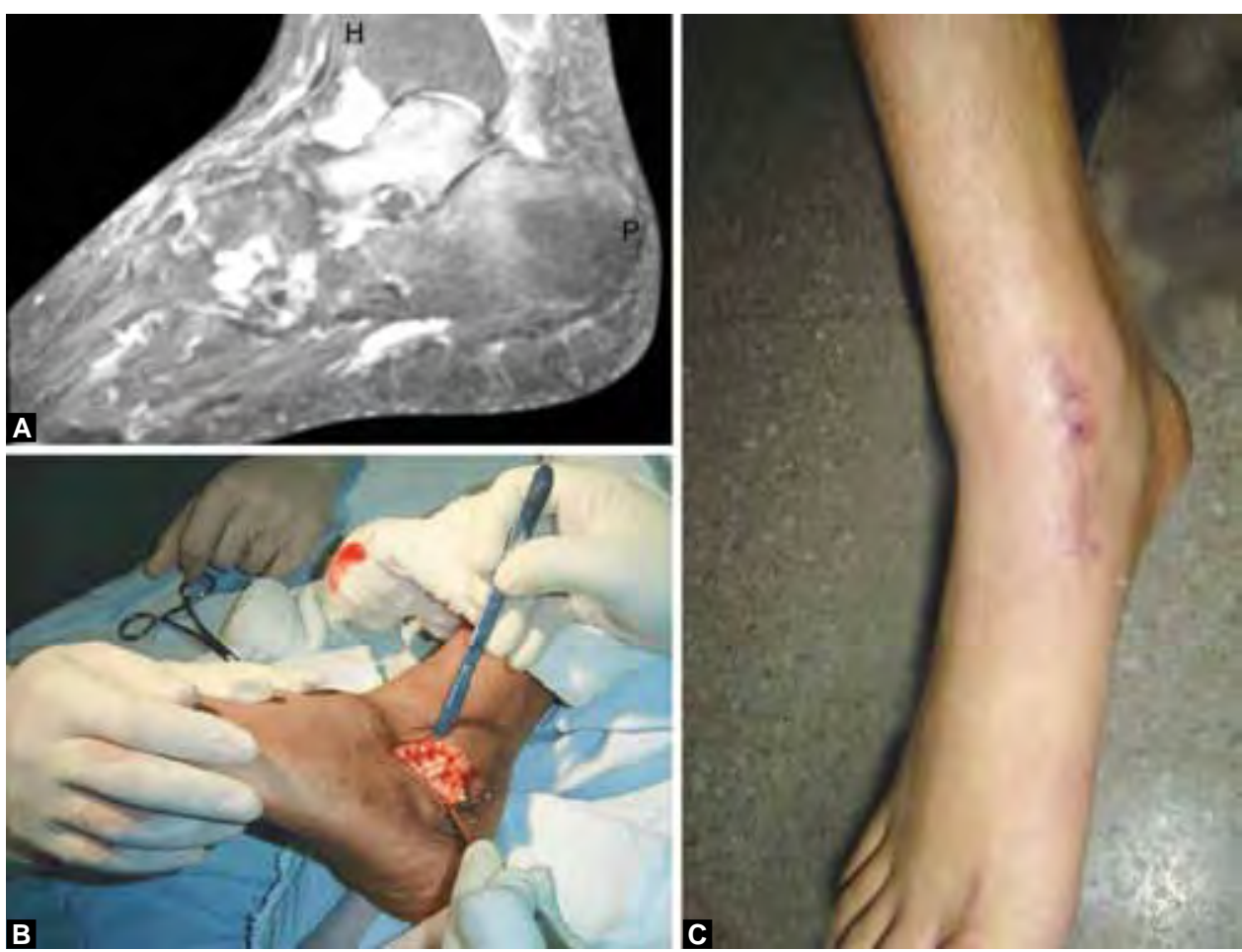

Figs 8A to C: Fungal granuloma of talus in a 27-year-old female: (A) Preoperative MRI showing hyperintense lesion within the talus, (B) intraoperative picture showing excision of the fungal granuloma and $(\mathrm{C})$ postoperative picture showing well healed wound after suture removal

of calcaneum treated with radical debridement, free partial medial rectus abdominis muscle flap coverage, transport distraction osteogenesis and novel antifungal therapy (combination of voriconazole and terbinafine). They reported good prognosis for a condition which historically required $100 \%$ amputation.

\section{TREATMENT AND REHABILITATION}

The primary management strategy involves appropriate culture specific antibiotics, management of the underlying systemic condition and surgical intervention, if required, with least soft tissue compromise. It is very important to time and plan the surgical approach for optimum results. Recent advances leading to the discovery of novel antimicrobial drugs and the emergence of multimodal treatment approach have created a paradigm shift in the management of hindfoot infections from amputation toward limb salvage. The advances and techniques have refined tremendously that now we try to attain premorbid status and full functional recovery in these conditions, which were initially thought incurable. ${ }^{17}$

Initially, amputation was the only solution for intractable hindfoot infections known to recur repeatedly resulting in disabling morbidity and at times mortality. Diabetes and other conditions resulting in immunocompromised states confound and worsen the condition. The saying 'infection burns in the fire of debridement' very well applies here and thorough radical debridement forms the basic step on which other treatment modalities are based. Any advanced treatment modality becomes ineffective in the scenario of inadequate debridement. Walsh et $\mathrm{al}^{18}$ had concluded that calcanectomy is an useful procedure for limb salvage in the presence of calcaneal osteomyelitis. Schade ${ }^{19}$ had also concluded that partial or total calcanectomy as a promising limb salvageprocedure. Similarly Kolker, ${ }^{20}$ Rochman, ${ }^{21}$ Saltzman ${ }^{22}$ and $\mathrm{Klos}^{23}$ reported successful limb salvage with talectomy followed by tibiocalcaneal arthrodesis using Ilizarov circular external fixator and other modalities respectively.

Recently the dead space management following radical debridement has seen a lot of advances in the form of autograft, tissue transfers as well as innovative spacers with ability to elude antimicrobials also. Endo et $\mathrm{al}^{24}$ described a staged hindfoot reconstruction using vascularized fibula graft in a patient with calcaneal osteomyelitis caused by methicillin resistant Staphylococcus aureus. Jeng et $\mathrm{al}^{25}$ and Loder et $\mathrm{al}^{26}$ had described the use of femoral head allograft for salvage of large defects following radical debridement of hindfoot infections. Papagelopoulos ${ }^{27}$ described a woman with Pseudomonas aeruginosa calcaneal osteomyelitis treated with debridement and PMMA beads with tobramycin and sequentially tobramycin impregnated calcium sulphate pellets and paste along with iliac crest autograft with successful outcome. Soft tissue coverage techniques have also advanced tremendously, with rotatory flaps as well as free flap techniques (Figs 9A to C). Panagakos et $\mathrm{al}^{28}$ 

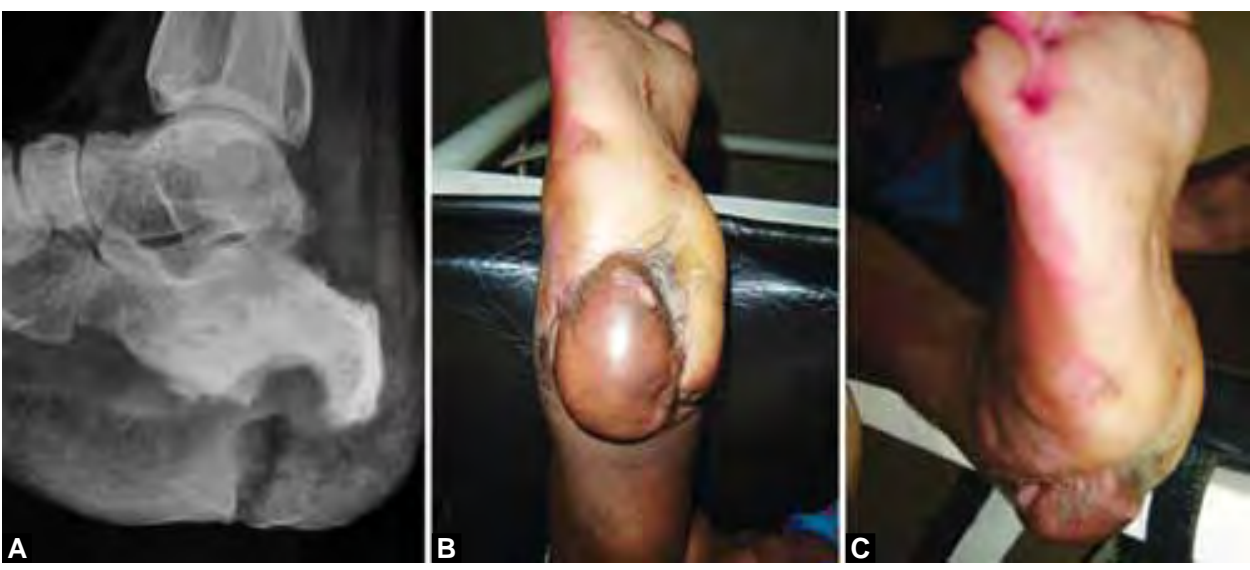

Figs 9A to C: (A) Radiograph of a patient with persistent osteomyelitis even after multiple debridements, (B and $C$ ) clinical photograph showing excellent results after calcanectomy and free heel flap

described de-epithelialized fasciocutaneous turnover flap for recurrent calcaneal wound with osteomyelitis.

Additionally, bone transport techniques employing distraction osteogenesis and pedicled free flaps can be used to improve the vascularity and hence, the outcome in these cases. Recently platelet rich plasma with properties of stypsis was used and had proven to be the preferred organic product for the treatment of chronic infections with soft tissue defects. ${ }^{29}$ The negative pressure wound management with VAC has proven to be a boon in management of infections with soft tissue defects and is presently being used frequently for hindfoot infections. ${ }^{30}$ Vacuum assisted closure (VAC) or negative pressure wound therapy (NPWT) is another efficient modality for both controlling infection and dead space management. The size of the wound defect may considerably diminish after VAC therapy, thus obviating the need for a larger wound cover.

\section{PROGNOSTIC FACTORS}

Merlet et al in their observation found that an American Society of Anesthesiologists (ASA) score $<2$, post-traumatic calcaneal osteomyelitis, age $<65$ years, absence of neuropathy, and absence of diabetes mellitus are good prognostic factors for healing without the need for amputation. ${ }^{1}$

\section{CONCLUSION}

Hindfoot infections can be devastating orthopedic problems, occurring from pyogenic spread or local infiltration after trauma or surgery. Early diagnosis and adequate management can help in reducing morbidity and attain good functional status. Tuberculosis can be treated by medical management, while pyogenic infections require adequate radical debridement, advanced dead space management techniques, synergistic systemic antimicrobial therapy and management of underlying systemic condition if any. Specific causes like thorn prick and foreign body granulomas, fungal infections, etc. have to be kept in mind. Once limb and life-threatening condition can be tackled with a timely multidisciplinary approach; the role of the plastic surgeon to provide appropriate flaps is pivotal.

\section{REFERENCES}

1. Merlet A, Cazanave C, Dauchy FA, Dutronc H, Casoli V, Chauveaux D, et al. Prognostic factors of calcaneal osteomyelitis. Scand J Infect Dis 2014;46(8):555-560.

2. Dhillon MS, Tuli SM. Osteoarticular tuberculosis of the foot and ankle. Foot Ankle Int 2001;22(8):679-686.

3. Fleming L, Ng A, Paden M, Stone P, Kruse D. Fungal osteomyelitis of calcaneus due to Candida albicans: a case report. J Foot Ankle Surg 2012;51(2):212-214.

4. Suresh SS, Zaki H, Shalamzari JE, Bhatnagar G. Osteomyelitis calcaneum due to a scorpion sting. J Foot Ankle Surg 2014; 53(3):340-343.

5. Jaakkola J, Kehl D. Haematogenous calcaneal osteomyelitis in children. J Pediatr Orthop 1999;19(6):699-704.

6. Carlson RM, Dux K. Shewanella putrefaciens, a rare cause of osteomyelitis. Int J Low Extrem Wounds 2013;12(3):231-233.

7. Gale DW, Scott R. Puncture wound of the foot? Persistent pain? Think of pseudomonas aeroginosa osteomyelitis. Injury 1991;22(5):427-428.

8. Yüksel S, Yüksel G, Oncel S, Divanli E. Osteomyelitis of the calcaneus in the newborn: an ongoing complication of Guthrie test. Eur J Pediatr 2007;166(5):503-504.

9. Wronka KS, Sinha A. Calcaneal osteomyelitis following steroid injection for plantar fasciitis: a case report. Foot Ankle Spec 2012;5(4):253-255.

10. Dhillon MS, Parsanna V, Goni V, Nagi ON. Wooden splinter induced pseudo tumour of the metatarsal. Foot Ankle Surg 2000;6(1):45-48.

11. Cracchiolo A. Wooden foreign bodies in the foot. Am J Surg 1980;140(4):585-587.

12. Dhillon MS, Nagi ON. Tuberculosis of the foot and ankle. Clin Orthop Relat Res 2002;398:107-113.

13. Dhillon MS, Aggarwal S, Prabhakar S, Bachhal V. Tuberculosis of the foot: an osteolytic variety. Ind J Orthop 2012;46(2): 206-211.

14. Sharma AK, Wakhlu A, Chaturvedi V, Agarwal LD. Primary mycetoma of the calcaneum. Trop Doct 199;23(3):135-136. 
15. Pennekamp PH, Diedrich O, Zhou H, Kraft CN. Foot injury as a rare cause of scendosporiosis with fetal outcome. Unfallchirurg 2003;106(10):865-868.

16. Cetrulo CL Jr, Leto Barone AA, Jordan K, Chang DS, Louie K, Buntic RF, Brooks D. A multidisciplinary approach to the management of fungal osteomyelitis: current concepts in post-traumatic lower extremity reconstruction: a case report. Microsurgery 2001;32(2):144-147.

17. Bhattacharyya A, Jha AK, Kumar S, Bhattacharyya B. Outcome of different modalities of surgical management of chronic osteomyelitis of calcaneum. J Ind Med Assoc 2012; 110(11):825-826.

18. Walsh TP, Yates BJ. Calcanectomy: avoiding major amputation in the presence of calcaneal osteomyelitis: a case series. Foot (Edinb) 2013;23(4):130-135.

19. Schade VL. Partial or total calcanectomy as an alternative to below-the-knee amputation for limb salvage: a systematic review. J Am Pediatr Med Assoc 2012;102(5):396-405.

20. Kolker D, Wilson MG. Tibiocalcaneal arthrodesis after total talectomy for treatment of osteomyelits of the talus. Foot Ankle Int 2004;25(12):861-865.

21. Rochman R, Jackson Hutson J, Alade O. Tibiocalcaneal arthrodesis using the Ilizarov technique in the presence of bone loss and infection of the talus. Foot Ankle Int 2008;29(10): 1001-1008.

22. Saltzman CL. Salvage of diffuse ankle osteomyelitis by single-stage resection and circumferential frame compression arthrodesis. Iowa Orthop J 2005;25:47-52.

23. Klos K, Lange A, Matziolis G, Wagner A. Tibiocalcaneal arthrodesis with retrograde nails. Description of a hindfoot procedure after massive talus destruction. Orthopade 2013; 42(5):364-366, 368-370.

24. Endo J, Kuniyoshi K, Mochizuki M, Shimoyama K, Koyama T, Aiba A, et al. Two-staged hindfoot reconstruction with vascularized fibula graft for calcaneal osteomyelitis caused by methicillin-resistant staphylococcus aureus: a case report. Microsurgery 2013;33(3):232-235.

25. Jeng CL, Campbell JT, Tang EY, Cerrato RA, Myerson MS. Tibiotalocalcaneal arthrodesis with bulk femoral head allograft for salvage of large defects in the ankle. Foot Ankle Int 2013;34(9):1256-1266.

26. Loder BG, Dunn KW. Functional reconstruction of a calcaneal deficit due to osteomyelitis with femoral head allograft and tendon rebalance. Foot (Edinb) 2014;24(3):149-152.

27. Papagelopoulos PJ, Mavrogenis AF, Tsiodras S, Vlastou C, Giamarellou H, Soucacos PN. Calcium sulphate delivery system with tobramycin for the treatment of chronic calcanealosteomyelitis. J Int Med Res 2006;34(6):704-712.

28. Panagakos P, McDonald P, Norem N, Shapiro H, Boc SF, Mitra A. De-epithelialized fasciocutaneous turnover flap for recurrent calcaneal wound with osteomyelitis. J Foot Ankle Surg 2014;53(1):83-87.

29. Wang HF, Gao YS, Yuan T, Yu XW, Zhang CQ. Chronic calcaneal osteomyelitis associated with soft-tissue defect could be successfully treated with platelet-rich plasma: a case report. Int Wound J 2013;10(1):105-109.

30. Stannard JP, Volgas D, McGwin G, Stewart RL, Obremskey W, Moore $\mathrm{T}$, et al. Incisional negative pressure wound therapy after high-risk lower extremity fractures. J Orthop Trauma 2012;26(1):37-42. 